\title{
Using electronic resources to support dialogue in undergraduate small-group teaching: the ASTER project
}

\author{
Frances Condron \\ Humanities Computing Unit, University of Oxford \\ email: Frances.Condron@oucs.ox.ac.uk
}

Lcarning through dialogue is an important element of UK higher education, supported by tutorial, seminar and workshop classes. Since 1998, the ASTER project has been exploring how Information and Communication Technologies support learning in small groups (http:l/cti-psy.york. ac.uk/asterl). Electronic resources are developed and used in courses to support a wide range of learning needs, from delivery of content to interactive teaching tools and assessment. The manner in which they are integrated into a course dictates the extent to which they support and extend learning. The ASTER survey has identified the use of a range of new technologies to support learning through dialogue in a variety of contexts. Many of the uses are common across disciplines, though we have observed some differences in the range of tools used, and how they are implemented in and beyond the classroom. These differences are partly determined by the subject content of resources, and by the activities that ICT tools support. Another factor influencing this variation seems to be traditions of academic discourse. The findings suggest that educational technology needs to support both generic education practice, and the special needs of particular disciplines.

\section{Introduction}

Of all the learning experiences to which students are exposed in British universities, some of the most intensely demanding and rewarding are those where students and tutor engage in lengthy discussion. For many disciplines, tutorials, seminars and workshops have a special role in developing expertise in applying complex and abstract theories to rich, diverse bodies of material. The ability to present convincing arguments, both written and verbal, is a skill that undergraduates need to acquire. Discussion between tutors and students, be it in the form of feedback on assessed work, structured classes or ad hoc meetings, can help students to acquire a deep understanding of their subject, and the confidence to find their own paths 
through a discipline. In a climate of still-rising student numbers and reduced funding, teaching in small groups has increasingly come under pressure. While new technologies can be used to supplement small-group teaching, their application is often costly and problematic. Within the UK, the past fifteen years has seen much time and money devoted to creating new tools, tutorials and datasets (most clearly through the Teaching and Learning Technology Programme). However, evaluations have highlighted problems with these new resources, and many resources are not used beyond the departments in which they were created (HEFCE et al., 1999). Because of the limited success of many earlier educational technology projects, research efforts have now shifted to focus on the pedagogic and subjectspecific factors determining the effectiveness of new tools in improving student learning.

The ASTER project (Assisting Small-group Teaching through Electronic Resources, http://cti-psy.york.ac.uklaster/) is funded by the Higher Education Funding Council for England (HEFCE), and is one of the Teaching and Learning Technology Programme's (TLTP) phase 3 projects. ASTER is a collaboration between the Universities of Oxford, Surrey and York (lead site), and University College Northampton. Since autumn 1998, ASTER has been exploring how new technologies support staff and students to make better use of small-group teaching, be it in tutorials, seminars, workshops, laboratories or other teaching situations. It is multidisciplinary, covering archaeology, classics, history, English literature, mathematics in engineering and related disciplines, philosophy, physics, psychology and religion. We have reported on good practice and expertise in the use of ICT, based on an extensive literature survey, email and telephone questionnaires, and more recently, detailed case studies of current UK practice. The survey and case studies were undertaken to gather evidence of current good practice in supporting small-group teaching with ICT. Reports on these surveys are available (ASTER, 1999; ASTER, 2000), outlining the range of tools currently in use, and the contexts in which they are used to support small-group teaching. They range from course Web pages and online reading consulted outside the class, to computer-mediated communication supplementing face-to-face meetings. This article focuses on the evidence for ICT supporting dialogue - extended discussions between and within a group with the purpose of exploring a particular topic, identifying and understanding varied viewpoints, and possibly reaching consensus.

While some digital resources act as a medium through which dialogue occurs, the majority of the ASTER case studies document indirect support, for example by providing material for consultation prior to the class (such as online documents, collections on CD-ROM), or for remediation to ensure that students can express themselves in specialist language (such as multimedia tutorials on essential mathematics). There is some overlap in the tools used across disciplines, but there are also important variations. These variations obviously arise through the limited availability of resources for any given topic, but this is no explanation for the selective use of tools by individual disciplines. What we also seem to be seeing is differences in academic discourse manifesting themselves in the choice of tools to support it. Before looking at the survey findings in more detail, we need to explore the relationship between academic discourse and teaching and learning.

\section{Small-group teaching, disciplinary differences and dialogue}

There are several interconnected factors determining what and how electronic tools and resources can be employed to support learning through dialogue. Discussion classes are 
vital to support students' acquisition of subject expertise and jargon, and to engage in academic discourse, both verbal and written. However, opportunities for discussion between students and tutors are reliant on the balance of teaching methods (lectures, practicals, seminars and so on) used, and class size, both of which vary between disciplines and institutions. Electronic tools can support discussion, mediating between individuals, though previous experience in using tools either for research or teaching by students and tutors influences their perception of the value of these tools.

Seminars, tutorials, workshops and laboratories not only give feedback and opportunities to develop subject expertise, they are also a vital avenue for helping students to adjust to departmental culture, and the UK university system. Close contact with staff motivates students to engage with their discipline, and staff can support students to integrate into the department and collaborate with peers. Thomas argues that male and female students (and by implication staff) have contrasting experiences of adjusting to departmental life, and in situating themselves within a departmental culture (Thomas, 1990). Although efforts need to be made to accommodate multiple approaches to learning, research shows that students whose approaches to learning match those of their discipline tend to engage most readily in their subject (Kolb, 1981). Ramsden and Entwistle suggest that the ways in which teaching is organized and delivered in departments influences students' orientation to learning (Entwistle and Ramsden, 1983: 188). Becher (1989) goes further, arguing that the use of particular learning and teaching methods within any single discipline is not only because they meet the needs of that discipline, but also because of the traditions and expectations that have developed in the discipline. Becher's 'academic tribes' welcome new members, but they must adjust their behaviour to fit in.

The balance of teaching practices used varies between institutions and disciplines, and opportunities for close support may be limited. Smeby's survey of Norwegian universities found seminars to be a prominent feature of humanities, arts and social sciences teaching, though not for the hard and applied sciences (Smeby, 1996). Becher has similar findings, arguing that these disciplinary differences in the balance of teaching methods are, like research, international (Becher, 1994). Smeby uses Kyvik's division of academic language into codified and literary languages to explain differences in teaching practices across discjplines in Norwegian universities. He argues that codified language is a feature of hard and applied sciences (such as mathematics, and also music), to which students are formally introduced. Students need to have a good grasp of specialist language in order to communicate in their discipline. By contrast, in humanities and social sciences, academic language is more literate, and although formal, the rules of style and expression are rarely directly transmitted to students. Although in very broad categories, Kyvik's division of academic language can be utilized to explain differences in educational practices across disciplines.

It is not therefore surprising that so many electronic tools and resources are used to support the varied activities that take place in university small-group teaching. Resources are not only selected for their subject content: presentation styles and the activities that tools and resources support should complement teaching practices. As argued above, these practices are deeply embedded in traditions of teaching and learning, and it should not be surprising that they are reflected in the range of electronic tools and resources used by any discipline. Moreover, using ICT in teaching can introduce new ways of teaching/learning/assessment, 
and unless this is recognized and accommodated for, the ICT intervention is not likely to be successful. Research into human-computer interaction can be fruitfully applied to the study of computer use in teaching and learning. Developed by Vygotsky, activity theory explores the ways in which humans act and interact through tools and resources, and social and cultural factors influencing such activity. The key term is mediation - how tools are used to support action and to achieve objectives, and how these tools are perceived by their users. As the user becomes more experienced with particular tools, perception shifts away from tools as objects restricting actions, the user becomes less conscious of the tool and focuses on the end goal (Nardi, 1996, provides a useful introduction to activity theory and new technology). Applying the same argument to higher education, academics within disciplines with a long tradition of using computers for research view them as part of a suite of working tools and a means towards an end (for example, astronomy - it is not possible to teach or research astronomy without computers). Students are therefore taught topics through/with computers as it is the accepted method for researching these areas. In disciplines with a short or limited experience of working with computers, computers can be seen as a barrier or restricting factor for one's work, and viewed with suspicion.

The ASTER project attempts to pull together these areas of research in teaching practice and the use of new technologies. While the ASTER survey has not been able to gather information on teaching practices across entire institutions, or nationally for all departments in a given subject area, it has nevertheless revealed differences in teaching practices between disciplines. These differences are not clear cut; the number of ASTER case studies is very small, and our findings cannot be taken as representative of UK higher education. However, they raise issues that deserve further attention.

\section{The ASTER survey}

In 1999, ASTER undertook a series of surveys to get a picture of current and good practice in how new tools and electronic resources are being used to support small-group teaching. A questionnaire was sent out by email to thousands of academics in Britain and beyond; 40 academics were interviewed by telephone; 30 case studies were carried out in late 1999 and early 2000. Data for the case studies were gathered through structured interviews with academic staff, informal feedback from students, and class observation (where possible). The case studies cover a range of subjects: archaeology, art, chemistry, classics, engineering, English, mathematics, physics, psychology and theology. All report on the use of ICT in face-to-face teaching, and are supported by an extensive literature survey. The case studies and reports from the surveys are available from the ASTER Web site (ASTER 2000; http:/lcti-psy.york.ac.uklaster/). Table 1 summarizes the findings for science and humanities disciplines, and psychology.

Many tools and resources are used to support teaching in and beyond the classroom. They are selected not only for their subject coverage, but also because their presentation style conforms to or complements traditional teaching practices for the given subject. While the former is easily recognized by academics, they may not be so aware of how the latter influences their choice of resources.

These tools are being used individually and in combination. Our survey revealed that the majority are consulted outside the classroom, providing material for discussion in class. 
Table 1: Summary of ASTER's findings on the use of C\&IT to support small-group teaching

The Web is widely viewed as a source for reading material prior to classes in the arts and humanities, though rarely used in the sciences, and not extensively used in psychology. The Web as a digital library duplicates the traditional practice of directing students to reading prior to the class, the only difference being the mode of access.

Our survey found that some psychologists were using virtual learning environments to post essential reading and set tasks prior to face-to-face classes. When employed in this way, VLEs facilitate discussion by providing content and information, but only in the same way as preparatory reading, irrelevant of the medium.

Multimedia tutorials are used to ensure that students have sufficient skills to continue with the course - thus they are popular in physics and engineering, particularly for mathematics; language drillers support students in acquiring basic vocabulary and grammar. Students are directed to multimedia tutorials for independent study, freeing up face-to-face meetings for more advanced discussion. They equip students with specialist languages in order to participate in face-to-face classes - mathematics for science undergraduates, and grammar for basic language learners. However, the ASTER survey found very little use of multimedia tutorials in the arts and humanities. 
Computer-mediated communication, and the communications features of virtual learning environments, directly support dialogue. Although an essential tool for distance learning, CMC supports traditional education and research. Case studies documenting successful and problematic experiences with CMC abound (for example, McNaught et al., 1999; Duffy et al., 1996). The ASTER survey found that CMC was used fairly extensively in the arts and humanities and psychology (to host discussions and support group work), but less so for undergraduate teaching in the sciences.

Computer-aided assessment is used in the sciences, and in psychology, for formative assessment (and feedback where it is built in), and sometimes also for summative assessment. However, it is not popular in the arts and humanities, where assessment tends to demand extended prose.

Although the ASTER sample is small, these preliminary findings suggest that:

- The sciences and some parts of the arts and humanities use ICT tools explicitly to introduce students to codified language - mathematics, grammar - essential for participating in academic discourse, and computer-aided assessment to review students' knowledge and capabilities.

- While computer-mediated communication tools support discussion, they do not readily support exchange in codified language as the majority of CMC tools have been developed for the Latin alphabet.

- Discussion in text-based disciplines also uses specialist dialogue, though the rules are implicit rather than explicit. Students are exposed to academic discourse through reading (either online or in print), and discussions take place through computers as well as face-to-face.

- Psychology falls between the two extremes - Kyvik's division of academic discourse into two types assumes that academics need specialize in only one, though psychology spans both. Arguably discourse in most subjects includes a mix of codified and literary language, and several tools may be required to support discourse.

We can further explore these findings by looking at the educational objectives expressed by academics for using electronic resources (Table 2). Not surprisingly, disciplinary differences emerge.

\begin{tabular}{lll}
\hline Arts and humanities & Psychology & Sciences \\
\hline Develop ICT skills & Develop ICT skills & Develop ICT skills \\
Improve verbal, written & Improve verbal, written & Improve verbal, written \\
communication & communication & communication \\
Introduce and develop research & Introduce and develop research & Introduce and develop research \\
methods & methods & methods \\
Access to written and pictorial & Access to material - case studies & Remediation (mathematics) \\
sources & Work in teams & Essential skills and techniques \\
Develop critical reading & & Grasp concepts \\
& & Help with visualization \\
& & Analysis \\
\hline
\end{tabular}

Table 2: Educational objectives served by electronic resources 
These differences are partly obscured by academics using the same terms to describe particular needs. For example, the range of ICT skills that students must acquire for a physics course is very different from those required for an English degree. However, there is some overlap in that all students need to be information literate. The drive, via the QAA and employers, for universities to instill key skills in students means that they should be able to communicate electronically as well as verbally and in writing. The above discussion focused on differences in academic discourse and how they are reflected in the range of tools used to support students in acquiring expertise and engaging with their subject. This suggests that generic tools such as email or text-based discussion lists, supporting literary language, do not serve learning and discussion in all disciplines equally.

Undergraduate degrees should equip students with the skills and knowledge to continue into research, should they choose to do so, and these are of course particular to the discipline. There is some variation in the sources of reading to support this research. While academic articles online are common to all subjects (often at a cost), arts and humanities undergraduate teaching benefits greatly from the many primary sources now available through the Web, and image-rich multimedia pages on topics that provide an overview of a subject which may otherwise be taught through texts. Access to materials for psychologists may be via CD-ROM, as case studies, used in a problem-based learning context where students are introduced to psychological methods and theories. In the sciences, the Web is not viewed as an important source of material for undergraduate teaching. However, simulations are valuable in helping students to understand concepts.

\section{Conclusion}

This brief review shows how the needs of disciplines are supported by particular electronic resources, both for the subject matter they contain, and for the teaching and learning activities that they support. The differences the ASTER survey has identified in the use of educational technology between disciplines is a manifestation of different traditions of teaching and learning, of varied uptake of computers, and the perceived value of ICT for resenrch and teaching. There is a genuine argument for educational technology to be sensitive to discipline needs in its pedagogic design as well as content. Generic tools such as virtual learning environments and computer-mediated communication support particular styles of dialogue and interaction which are not common or of equal value to all disciplines. Subject-specific tools and resources - online resources, simulations and multimedia tutorials - address particular teaching and learning needs. There is no definitive good practice in supporting dialogue through electronic resources. Rather, good practice comes about through matching teaching and learning activities to the individual tools or resources that support them. Where new activities are introduced by the tools, additional support may well be required to overcome the many barriers to change.

\section{Acknowledgements}

This article builds on work carried out by the ASTER team: Dick Bacon, Catherine Bennett, Chris Colbourn, Angela Fisher, Michael Fraser, Nick Hammond, Sylvia Hogarth, Anne Jelfs, Stuart Lee, Simone Richardson, Lee Sproats, Lena Tostevin, Annie Trapp, David Wonnacott and the author. 


\section{References}

ASTER (1999), 'A survey of current practice in the use of C\&IT to support small-group teaching activities in disciplines associated with the humanities, physics, and psychology', York: ASTER, http://cti-psy.york.ac.uklaster/publications/Report_2/report_2. html.

ASTER (2000), 'Investigating the use of electronic resources in small-group learning and teaching', York: ASTER [http://cti-psy.york.ac.uk/aster/resources/publications/csreport/ csreport.html].

Becher, T. (1989), Academic Tribes and Territories: Intellectual Enquiry and the Cultures of Disciplines, Milton Keynes: SRHE/Open University Press.

Becher, T. (1994), 'The significance of disciplinary differences', Studies in Higher Education, 19, 151-61.

Entwistle, N. J. and Ramsden, P. (1983), Understanding Student Learning, London: Croom Helm.

Hannan, A., English, S. and Silver, H. (1999), 'Why innovate? Some preliminary findings from a research project in teaching and learning in higher education', Studies in Higher Education, 24, 279-89.

HEFCE, HEFCW, SHEFC, DENI et al. (1999), Communications and Information Technology Materials for Learning and Teaching in UK Further and Higher Education, Report 99/60, NISS.

Laurillard, D. (1993), Rethinking University Teaching, London: Routledge.

Nardi, B. A. (ed.) (1996), Context and Consciousness: Activity Theory and HumanComputer Interaction, Cambridge, Mass: MIT Press.

Ramsden, P. (1992), Learning to Teach in Higher Education, London: Routledge.

Smeby, J. C. (1996), 'Disciplinary differences in university teaching', Studies in Higher Education, 21, 69-79.

Stoner, G. (ed.) (1996), Implementing Learning Technology, Edinburgh: Learning Technology Dissemination Initiative, http://www.icbl.hw.acuk/ltdilimplementing-it/cont.htm (last accessed 3 September 2000).

Thomas, K. (1990), Gender and Subject in Higher Education, Buckingham: SRHE/Open University Press. 